\section{The Uppsala Conference of the HEPP Division}

\author{
Guido Altarelli, CERN
}

The International High Energy Physics Conference (HEP 87) organized by the HEPP board of EPS was held at Uppsala in Sweden from June 25 to July 1, 1987. The conference was attended by 600 physicists who came to Sweden's oldest university, founded 510 years ago, from 33 countries all over the world. The city of Uppsala, blessed by reasonably good weather during the conference days (with practically 24 hours a day of sunlight), revealed itself as an ideal place for a conference of this size. In fact Uppsala, being a university town, offers many convenient facilities in a quiet surrounding. The nearby city of Stockholm and the beautiful Swedish countryside added the necessary contour of touristic attraction. The merit of a remarkably good organization goes mainly to Prof. R. Salmeron, Chairman of the HEPP Board of EPS, to Prof. S. Kullander, Chairman of the local Organizing Committee, and to Prof. T. Lindqvist. The hospitality of the local community of physicists was splendid and I personally could appreciate their kindness and the elegant warmth of their houses.

Over the past year, the most important development in physics and the most spectacular phenomenon have occurred outside the domain of particle physics (although rich in consequence for it). High temperature superconductivity was reviewed for the Conference by $\mathrm{G}$. Bednorz from IBM-Zurich, while the implications for particle physics of the 1987 supernova explosion were discussed by $A$. de Rujula and also by $M$. Roos, J. Ellis and B. Degrange.

Within the specific field of particle physics, the present year was mainly one of consolidation while waiting for the results of the new experiments which are in a more or less advanced state of preparation. The parameters of the main high energy machines are listed in Table 1. In particular at Uppsala the first physics results from two new accelerators were presented. From Tristan, the $\mathrm{e}^{+} \mathrm{e}^{-}$Japanese collider with centre of mass energy $\sqrt{ } s=50 \mathrm{GeV}$, we heard from A. Bodek and T. Kondo that the hadronic events collected by the Tristan detectors Venus, Topaz and Amy are consistent with five flavours of quarks, so that the limit on the top quark mass can now, or will soon be displaced from $23 \mathrm{GeV}$ (Petra) to $25 \mathrm{GeV}$. From Fermilab, L. Halloway presented the evidence for the first American-born intermediate vector bosons $\mathrm{W}$ and $\mathrm{Z}$, produced by the Tevatron working at $\sqrt{ } s=1.6 \mathrm{TeV}$.

The future development of particle physics depends very much on the progress of accelerators and detectors. Two plenary sessions were devoted to new ideas on particle acceleration (T. Weiland) and to developments in detector technology (T. Ypsilantis).

At the Conference, the standard model of strong and electroweak interactions was further consolidated, as discussed among others by P. Darriulat, $\mathrm{K}$.

Table - STATUS OF ACCELERATOR PROJECTS

\begin{tabular}{llccccc}
\hline Location & Name & $\begin{array}{c}\text { Length } \\
(\mathrm{km})\end{array}$ & Particle & $\begin{array}{c}\text { Beam Energy } \\
(\mathrm{GeV})\end{array}$ & $\begin{array}{c}\text { Luminosity } \\
\left(\mathrm{cm}^{-2} / \mathrm{s}\right)\end{array}$ & Running \\
Beijing, China & BEPC & 0.24 & $\mathrm{e}^{+}+\mathrm{e}^{-}$ & 2.8 & $(3-17) \times 10^{30}$ & $1989-92$ \\
CERN, Europe & LEP 1 & 27 & $\mathrm{e}^{+}+\mathrm{e}^{-}$ & 55 & $1.6 \times 10^{31}$ & 1989 \\
& LEP 2 & 27 & $\mathrm{e}^{+}+\mathrm{e}^{-}$ & 95 & $2.8 \times 10^{31}$ & 1993 \\
& SPS/ACOL & 7 & $\bar{p}^{-}+\mathrm{p}$ & 315 & $4 \times 10^{30}$ & 1987 \\
Hamburg, FRG & HERA & 6.3 & $\mathrm{e}+\mathrm{p}$ & $30+820$ & $2 \times 10^{31}$ & 1990 \\
Serpukhov, USSR & UNK 1 & 20 & $\mathrm{p}$ & 3000 & & 1990 \\
& UNK 2 & 20 & $\mathrm{p}+\mathrm{p}$ & 3000 & & 1993 \\
Tsukuba, Japan & TRISTAN & 3.2 & $\mathrm{e}^{+}+\mathrm{e}^{-}$ & 25 & $6 \times 10^{30}$ & 1987 \\
Fermilab, USA & TEVATRON & 7 & $\overline{\mathrm{p}}+\mathrm{p}$ & 900 & $1.2 \times 10^{29}$ & 1987 \\
Stanford, USA & SLC & & $\mathrm{e}^{+}+\mathrm{e}^{-}$ & 51 & $6 \times 10^{27}-6 \times 10^{30}$ & $1987-90$ \\
USA & SSC & 83 & $\mathrm{p}+\mathrm{p}$ & 20000 & $10^{33}$ & $1995-96$ \\
\hline
\end{tabular}

The SSC is so far approved by the U.S. President. In addition to these projects, a large hadron collider (LHC) to be placed in the LEP tunnel has been proposed by CERN. It would by the mid 90 s generate collisions between protons of $8 \mathrm{TeV}$ and between $50 \mathrm{GeV}$ electrons and $8 \mathrm{TeV}$ protons.

\title{
APPLICATIONS
}

Third in the Series

21 papers, 341 pages

ISBN 0-89252-683-1

Copyright 1987

Editor: James Chang

Kaman Sciences Corporation 7-11 April 1986

Howey-in-the-Hills, Florida USA

The concept of the SPIE Institutes for Advanced Optical Technologies developed out of SPIE's desire to foster increased interaction and collaboration among researchers working in emerging optical technologies. This volume is a result of that interaction.

Part One

Photonic Recorders and Systems

2 papers

Part Two

Component Technologies

5 papers

Part Three

Photonic Sensors

11 papers

Part Four

Nonlinear Effects

3 papers

\section{Contributors}

Gary R. Allen - Robert F. Benjamin

J.M. Bernet • Michel Bouchu $\bullet Y$. Bourbin George 1. Chandler - James Chang

Gordon W. Day • E. D, Humieres • P. Girard

C. Imhoff $\bullet$ N.S.P.King $\bullet$ Kam Y. Lau Jacques G. Lefebvre $\bullet$ Cheng-Huei Lin Mark E. Lowry $\bullet$ Peter B. Lyons GlenM. McWright - Melvin A. Nelson Barry T.Neyer - James W. Ogle $\bullet$ M. Papuchon

C.Puech - Robert P. Reedy - Frank Roeske Mark D. Rotter - R. Clayton Smith

Vern N. Smiley $\bullet$ K.Tai $\bullet$ Douglas R. Thaye

A. Tomita $-S$. Vatoux $\bullet$ Lynn R. Veese Bruce M. Whitcomb - Mark Wilke

SPIE Member $\$ 54$

Nonmember North America $\$ 65$ Nonmember All Other Countries \$74

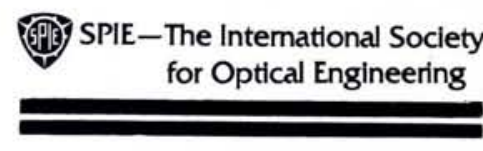

To order: contact SPIE, Avenue de la Tanche 2, B-1160 Brussels, Belgium; Telephone 2/674.52.12; Telex 25387 AVVAL B. In North America contact SPIE, P.O. Box 206/676-3290:Telex 46-7053. 
Schubert and B. Degrange and indications of possible deviations from present orthodoxy were eliminated or at least substantially reduced by improved experiments.

One of the main "leit-motiven" of the Uppsala conference was the quest for experimental information on the mass $m_{t}$ of the top quark, the sixth flavour, yet to be observed, that must necessarily exist for the standard model to be consistent. Several experimental results have recently contributed to restrict further the range of possible values. Whilst the most trustworthy lower bounds on $m_{t}$ are from the $\mathrm{e}^{+} \mathrm{e}^{-}$experiments of Petra and Tristan $\left(m_{\mathrm{t}}>25 \mathrm{GeV}\right)$ the UA1 collaboration at CERN presented a new limit $m_{t}>44 \mathrm{GeV}$ ( $95 \%$ c.l.). This was obtained from the study of charged lepton signals (electrons and muons) in proton anti-proton collisions at $\sqrt{ } s=0.63$ $\mathrm{TeV}$ at the Spps collider at CERN. The main assumptions in the derivation of this limit (which supersedes a previous indication of a positive signal by the same group) are that the semileptonic branching ratio of the top quark is "normal" and QCD-based predictions for the production rate and the decay momentum distributions are correct. These assumptions being relatively mild, the UA1 bound is a very important result.

A completely different indication for a heavy top quark is obtained from the recent measurement by the ARGUS collaboration at DESY of a surprisingly large amount of mixing between the beautyflavoured mesons $\mathrm{B}_{\mathrm{d}}^{\circ}$ and $\overline{\mathrm{B}}_{\mathrm{d}}^{\circ}$ composed of $a$ b $\bar{d}$ or $\bar{b} d$ pair of quark-antiquark respectively. This experiment can be awarded the title of "experiment of the year". It has prompted a flurry of theoretical papers not far from that originated by the supernova explosion. The general conclusion is that within the standard model, if one assumes three generations of quarks and leptons and reasonably conservative values for a combination of unmeasured parameters, then the top quark mass is rather safely bound by $m_{t}$ $>45 \mathrm{GeV}$. Indeed, if the data are taken at face value, the most likely location for the top quark mass would be in the range $90 \mathrm{GeV}<m_{\text {, }}<150 \mathrm{GeV}$. However the presence of a fourth family of quarks and leptons, not yet excluded by experiment, would make the connection between the ARGUS result and the top quark mass completely disappear.

On the other hand, upper limits on the top quark mass can be obtained from experimental tests of the electroweak theory. These limits are derived in the following way. The most precise determinations of $\sin ^{2} \theta_{w}$, with $\theta_{w}$ being the weak angle, are obtained from the value of the $\mathrm{W}$ mass, measured by the UA1 and UA2 collaborations at CERN, and from neutrino-nucleon deep inelastic scattering (the most accurate results being those of the CDHS and CHARM experiments at CERN and CCFFR at Fermilab). The two values of $\sin ^{2} \theta_{w}$ agree within the experimental errors only if a sizable contribution from the electroweak radiative corrections is included. These corrections have a sensitive dependence (quadratic) on the top quark mass $m_{\mathrm{t}}$. Agreement is only obtained for $m_{\mathrm{t}}<190-230 \mathrm{GeV}$. A comparable upper bound is also derived by comparing the experimental value of the $\rho$-parameter lobtained from the measured ratio of neutral to charged weak current effects) with the theoretical value, which again depends on radiative corrections.

Thus it now appears quite likely that the top quark is too heavy to be present (because of phase space) in Z-decay products. If so, which experiment will be able to discover it in the near future? In general, $\mathrm{e}^{+} \mathrm{e}^{-}$colliders are the most efficient quark discoverers, if their energy is enough for production. If indeed $2 m$, $>M_{Z}$, then the chances of SLC and LEP1 are small (although LEP1 is in a better position because it can reach a beam energy of $55 \mathrm{GeV}$ ). At LEP2 the top quark can only be observed if $m_{t}<100$ $\mathrm{GeV}$ (and only many years from now). It follows that the hadron colliders have the best chances in the near term. At the Spps collider of CERN, the increased luminosity obtained with $A C O L$ will allow the range $m_{t}<100 \mathrm{GeV}$ to be explored. Above this range there is only the Fermilab Tevatron with its larger centre of mass energy: $\sqrt{ } s=1.6 \div 2 \mathrm{TeV}$ in comparison with the Spps collider $(\sqrt{ } s$ $=0.63 \mathrm{TeV}$ ).

Another subject that received much attention at the Conference was the present limits on the number of quark and lepton families. These limits are derived by counting the number of existing neutrino species using different methods. From the observed abundances of light elements in the Universe (especially helium) one derives that at most two new species of light neutrinos, in addition to the known, are allowed. A numerically equivalent result is obtained by measuring the cross-section for $\mathrm{e}^{+} \mathrm{e}^{-}$ $\rightarrow \gamma+\Sigma v_{i} \bar{v}_{i}$ (i.e. photon and nothing else visible). According to the standard model, the observed rate is a sum of known contributions from each of the neutrino species produced in which case the counted neutrinos can be considerably heavier than in the previous.
Finally, from $p \bar{p}$ collider experiments the number of neutrinos that contribute to the $Z$ decay width can be derived from the observed ratio of $\mathrm{W} \rightarrow$ ev versus $\mathrm{Z} \rightarrow$ $\mathrm{e}^{+} \mathrm{e}^{-}$events (i.e. $\mathrm{p} \overline{\mathrm{p}} \rightarrow \mathrm{W}+$ anything, followed by $\mathrm{W} \rightarrow \mathrm{ev}$, and similarly for the $Z$ ). For that purpose one needs to compute the ratio of the $\mathrm{W}$ and $\mathrm{Z}$ production cross-sections by Quantum Chromodynamics and to obtain the total $\mathrm{W}$ width from the standard electro-weak theory (assuming that no new charged lepton is light enough to allow the decay $\mathrm{W} \rightarrow$ $\left.L v_{L}\right)$. It is interesting to remark that the resulting limit on the number of neutrinos depends in this case, in a substantial way, upon the top quark mass. In fact it is important to know if the decays $\mathrm{W} \rightarrow$ $\mathrm{tb}$ and $\mathrm{Z} \rightarrow \mathrm{tt}$ exist or not. If one only assumes $m_{\mathrm{t}}>45 \mathrm{GeV}$ then the result from combining the UA1 and UA 2 data is again that at most two extra neutrinos are allowed. But new families are only possible if the top quark is relatively light. In conclusion, the family repetition which is so peculiar and mysterious is not to continue for ever.

In the field of strong interactions interesting new results were also presented at the Uppsala conference. I found especially important the set of new results on the nucleon structure functions by the BCDMS collaboration at CERN. The impact of these new results on our knowledge of the parton densities in the nucleon, especially in connection with the d/u ratio, was much discussed. Also interesting are the results by the EMC group at CERN on the structure functions of polarized protons.

The continuous improvemet in the description of the process of hadronization, i.e. the transformation of quarks and gluons into jets of hadrons, was well reviewed by G. Gustafson from the Lund School (that has contributed so much to this subject).

Current progress in heavy ion physics was summarized by W. Willis who, in his balanced report, correctly stressed the importance of formulating and performing quantitative tests of thermalization in the collision products and of the presence of a mixed phase (the ordinary nuclear matter mixed with quark-gluon plasma). Experiments now in progress should probably produce decisive results by the next EPS conference (in Madrid 1989).

$\mathrm{J}$. Prentice defined the past year as a "vintage year" for hadron spectroscopy and reviewed the most recent additions to the long list of existing mesons and baryons. These days, newcomers must be somewhat exceptional to be noticed: for example a baryon carrying three 
units of strangeness $\Omega^{*}$ (2253), like the famous $\Omega(1642)$ that in the sixties established the SU(3)-symmetry among the $\mathrm{u}, \mathrm{d}$ and s light quarks, or a baryon carrying both charm and strangeness.

From time to time it is good to interrupt a long series of experimental results by listening to a pure theoretical talk. The eminent Russian field theorist L.D. Faddeev offered a very clear and solid seminar on a possible strategy toward giving sense to theories with anomalies.

The fascinating interplay between particle physics and cosmology was described by J. Ellis. He stressed with special emphasis the importance of obtaining an experimental solution to the problem of dark matter in the Universe (see page 138). An exciting possibility discussed by Ellis is that the mysterious all pervading substance could be made of photinos, the supersymmetric partners of the ordinary photons. Supersymmetry at relatively low energy was also discussed by $\mathrm{G}$. Kane in his report on the possible avenues beyond the standard model. While the relevance of supersymmetry for physics at energies accessible now or in the predictable future in our laboratories is at the centre of the debate, many theorists think that supersymmetry can be considered as established at the Planck mass. This belief arises from the, at present, most promising framework for a theory of quantum gravity, namely the theory of superstrings, reviewed by M.B. Green. As perhaps one could have anticipated, there is now less optimism about any shortrange practical feedbacks into particle physics of this really gigantic conceptual enterprise.

I think that a very lively and healthy picture of particle physics has emerged once more at the Uppsala conference. In this respect the formula with both parallel and plenary sessions has confirmed its virtues. Although perhaps more demanding for the audience it has the merit of offering a larger number of facets of the field. The community of particle physics is at present working very hard to explore better the foundations of the standard model hoping to find a clue toward a new layer of physics. In this respect, as I tried to make clear in my summary talk, the most important goal of particle physics in the next decade is to clarify experimentally the origin of the Fermi scale of mass, of order $G_{F}^{-1 / 2} \cong 300 \mathrm{GeV}$ which fixes the $W$ and $Z$ masses. In other words, one has to validate the Higgs sector of the electroweak theory, which is responsible for the spontaneous breaking of the gauge symmetry. There are, in fact,

\section{Experimental
Nuclear Physicist}

There is a vacancy for an experimental physicist to join the Nuclear Structure Facility (NSF). The experimental group is involved in carrying out and supporting a broadly based research programme using a $20 \mathrm{MV}$ tandem.

The successful applicant will liaise with university research teams, collaborate in nuclear research programmes and play an active role in initiating and developing new programmes. Other duties will involve work on the design and development of major equipment through to commissioning, operation and maintenance. As a member of the in-house team of scientists he/she will be expected to provide expertise in the methods of experimental nuclear physics and to aid and direct the technical and scientific support staff in operating the NSF and for diagnosing problems as they arise.

Applicants should have a good honours degree in an appropriate discipline or equivalent with a period of postgraduate experience. $\bar{A} \mathrm{Ph} . \mathrm{D}$ degree in nuclear physics and a period of post doctoral experience in experimental nuclear physics would be an advantage. The appointment will be in the grade of Higher Scientific Officer with a salary range of $£ 9219$ to $£ 12505$, starting salary depending on qualifications and experience. The superannuation scheme is non-contributory.

CLOSING DATE - 15 December 1987.

For further information please contact: Dr. J. S. Lilley on (England) 0925 (603558). Application forms may be obtained quoting reference DL/26 from: The Personnel Officer, Science and Engineering Research Council, Daresbury, Warrington WA4 4AD, England. (24 hour answering service). convincing theoretical arguments indicating that some form of new physics must be hidden near the Fermi scale. In particular, a fundamental Higgs appears to require supersymmetry signals just above the Fermi scale. Alternatively, some form of compositeness should become manifest, or possibly even supersymmetry and compositeness. Whatever the final outcome, it seems unavoidable that experimental studies in the $\mathrm{TeV}$ energy domain will lead to new fundamental discoveries. The large effort which is being made precisely aims at crossing this new frontier of particle physics.

\section{Nobel Prize for Physics}

Following closely on the award of the 1988 EPS Hewlett-Packard Europhysics Prize, the 1987 Nobel Prize for Physics has been awarded to J.G. Bednorz and K.A. Müller of the Zürich Laboratory of IBM for their discovery of high temperature superconductivity in a layered oxide of copper, barium and lanthanum.

An appreciation of their work will be given in the next issue of Europhysics News.

\section{physics. \\ -

\section{Postdoctoral Positions}

The Institute for Studies in Interface Sciences (ISIS) of the University of Namur has several research positions at the postdoc level for physicists or chemists to work in the following areas:

1) STM microscopy and spectroscopy:

2) Thin film synthesis and interface characterisations;

3) Theoretical research in physico-chemical properties of inter facial systems. The one-year positions are renewable and available immediately.

Applications should reach Prof. A. Lucas, ISIS-FUNDP,

61, rue de Bruxelles, B - 5000 Namur, Belgium.

Tel. (32) (81) 229061. 